\section{Triamcinolone Acetonide Mesotherapy in the Treatment of Recalcitrant Patches of Alopecia Areata - A Pilot Study}

\section{Chandrashekar BS*, Sandeep MA, Vani Vasanth, Jagadish P and Rajashekar ML}

CUTIS, Academy of Cutaneous Sciences, Bengaluru, Karnataka, India

\begin{abstract}
Background

Alopecia areata is a recurrent, chronic, socially stigmatising non scarring autoimmune disease in which patchy hair loss occurs in some or all areas of the body. It is estimated to be the presenting complaint in $2 \%$ of dermatologic consultations. Various modalities of treatment have been tried with variable outcomes.

Aim

To study the effect of therapeutic wounding by triamcinolone acetonide mesotherapy on hair growth in patients suffering from alopecia areata.

\section{Methods}

Fifteen patients of alopecia areata, who had tried various modalities of treatment beforehand (including topical and systemic corticosteroids) were recruited in the study. Of the 15 subjects, 4 had Alopecia totalis, 4 had ophiatis pattern of AA, 6 had discrete patches and 1 had diffuse pattern of AA. Five sessions of treatment with triamcinolone acetonide delivered to the scalp by microneedling, was done at an interval of 3 weeks each. Subjective and objective assessment was done after 3 months.

Result

At the end of 3 sessions itself, there was significant improvement in the treated areas in 8 of 15 patients with appearance of numerous vellus hair and gradual conversion to terminal hair at the end of treatment. After 6 sessions, the number of patients who experienced the improvement increased to 12 .

Conclusion

Therapeutic wounding procedure can be effectively performed at the affected site in patients suffering from alopecia areata.
\end{abstract}

"Corresponding author: Chandrashekar BS, CUTIS, Academy of Cutaneous Sciences, Bengaluru 560040, Karnataka, India, Tel: +91 9740091155; E-mail: cutisclinic@gmail.com

Citation: Chandrashekar BS, Sandeep MA, Vani Vasanth, Jagadish P, Rajashekar ML (2015) Triamcinolone Acetonide Mesotherapy in the Treatment of Recalcitrant Patches of Alopecia Areata - A Pilot Study. $\mathrm{J}$ Clin Dermatol Ther 2: 006

Received: December 24, 2014; Accepted: February 04, 2015; Published: February 18, 2015
Keywords: Alopecia areata; Hair growth; Mesotherapy; TMA Rolling

\section{Introduction}

Alopecia Areata (AA) is an autoimmune and chronic disease that is difficult to treat. It is estimated to be the presenting complaint in $0.7 \%$ of new dermatologic consultations in India [1].

Topical treatments in alopecia areata aim to achieve hair regrowth, but do not alter the underlying pathology, whereas systemic treatments can modify the course of the disease. In neither case does treatment provide a cure [2,3]. Corticosteroids either intralesionally or topically remain the first line of treatment [4].

A few cases of alopecia areata are resistant to intralesional steroid therapy and are often difficult to treat. This may be due to a need of larger doses and often accompanied by pain while giving them. Microneedling with dermaroller cause small pinpoint wounds. Advancements in molecular research have led to the identification of numerous proteins and genes which get stimulated on wounding, namely vascular endothelial growth factor, $\boldsymbol{\beta}$-catenin, Wnt3a and Wnt3b [5]. Therefore we attempted a pilot study to assess the efficacy of combiningtherapeutic wounding (microneedling) along with Triamcinolone Acetonide Mesotherapy (TMA) on recalcitrant alopecia areata.

\section{Materials and Methods}

A prospective pilot study of 15 patients was done at our institute between September 2012 and March 2014. The study population comprised of 10 males and 5 females, aged between 10-35 years. A detailed history was taken and relevant clinical examination was done. Various treatment options tried by the patients before coming to us included systemic \& topical corticosteroids, minoxidil ( $2 \%$ \& 5\%), immunotherapy and even alternative medicine. Inclusion criteria included those who had not achieved improvement or had worsened after atleast 3-4 months of strict adherence to the treatment given. A minimum of 6 months of therapy-free interval before coming to our institute was considered for inclusion so as to make sure that delayed response to the above mentioned therapies didn't interfere with the interpretation of our results. Patients were asked to trim their hair as short as possible. The affected areas were prepared by cleaning with acetone. $1 \mathrm{ml}$ of $10 \mathrm{mg} / \mathrm{ml}$ triamcinolone acetonide was applied drop by drop. A standard dermaroller having 192 needles of $0.5 \mathrm{~mm}$ length each was used, which resulted in formation of around 250 holes $/ \mathrm{cm}^{2}$ when rolled over an area for 15 times. The affected areas were gently and repeatedly stroked in horizontal, vertical and diagonal directions till petechiae or pin point bleeding developed. The procedure was practically painless and therefore no local anaesthesia was used. However, for those who didn't want to tolerate the pain, topical anaesthesia with $2 \%$ Lignocaine, under occlusion, was applied for 45 minutes prior to procedure. The procedure was repeated for six sessions with an interval of three weeks between consecutive sessions. Patients were instructed to continue applying $5 \%$ minoxidil on the patches once daily, after 24 hours of performing the dermaroller 
Citation: Chandrashekar BS, Sandeep MA, Vani Vasanth, Jagadish P, Rajashekar ML (2015) Triamcinolone Acetonide Mesotherapy in the Treatment of Recalcitrant Patches of Alopecia Areata - A Pilot Study. J Clin Dermatol Ther 2: 006.

treatment. During each visit, dermoscopic changes over a pre-selected site for each patient were noted.

The parameters employed for evaluation of efficacy were:

1. Clinician's assessment of improvement after 6 sittings

2. Patient's assessment of improvement after 6 sittings

\section{Clinician's assessment of improvement after 6 sittings}

Dermoscopic views of the affected area, using 3Gen Dermlite dermoscope, were photographed before commencement of mesotherapy with TMA and before the start of subsequent such sessions, for the appearance and distribution of vellus hair. The results were graded as depicted in table 1 .

\begin{tabular}{|c|l|}
\hline Grade & \multicolumn{1}{c|}{ Status } \\
\hline 0 & No vellus hair \\
\hline 1 & Few vellus hairs seen \\
\hline 2 & Significant vellus hair seen \\
\hline
\end{tabular}

Table 1: Grading of investigator's assessment.

\section{Patient's assessment of improvement after $\mathbf{6}$ sittings}

The patients were asked to assess their improvement as per the grading in table 2 .

\begin{tabular}{|c|l|}
\hline Grade & \multicolumn{1}{|c|}{ Status } \\
\hline 0 & No improvement \\
\hline 1 & Satisfactory improvement \\
\hline 2 & Good improvement \\
\hline 3 & Excellent improvement \\
\hline
\end{tabular}

Table 2: Grading of patient's improvement.

\section{Results}

From the third session itself, subjective and objective changes in the form of appearance of vellus hair (grade 1 and 2) confirmed by dermoscopy, were noticed in 8 of the 15 patients, the number increased to 12 patients at the end of six sessions. After the sixth session, patients were maintained on $5 \%$ topical minoxidil $(2 \%$ Minoxidil in Paediatric age group) and followed up after every two months for a period of 6 months. No relapse was noted in any of the patients. The results depicting the progress are shown in figures 1-4.

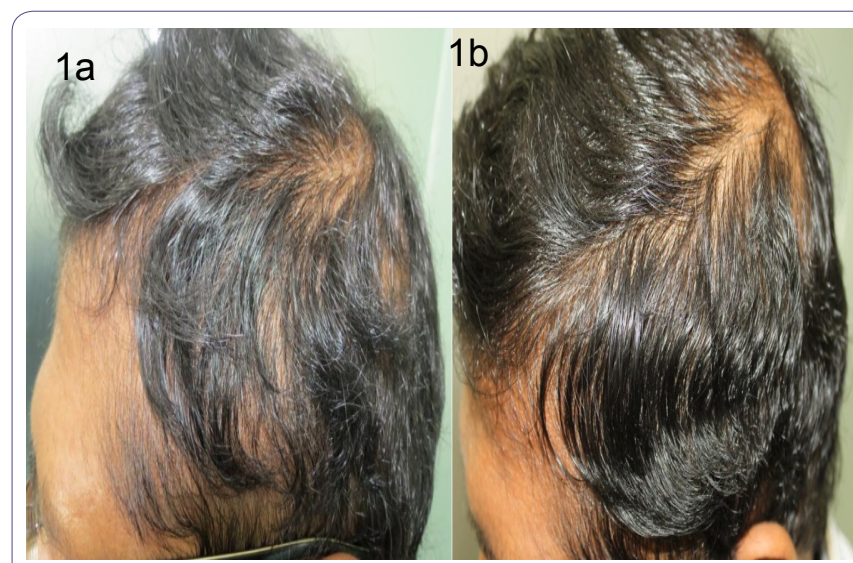

Figure 1: a) Diffuse pattern : At the start of treatment; b) After 6 sessions of TMA Mesotherapy

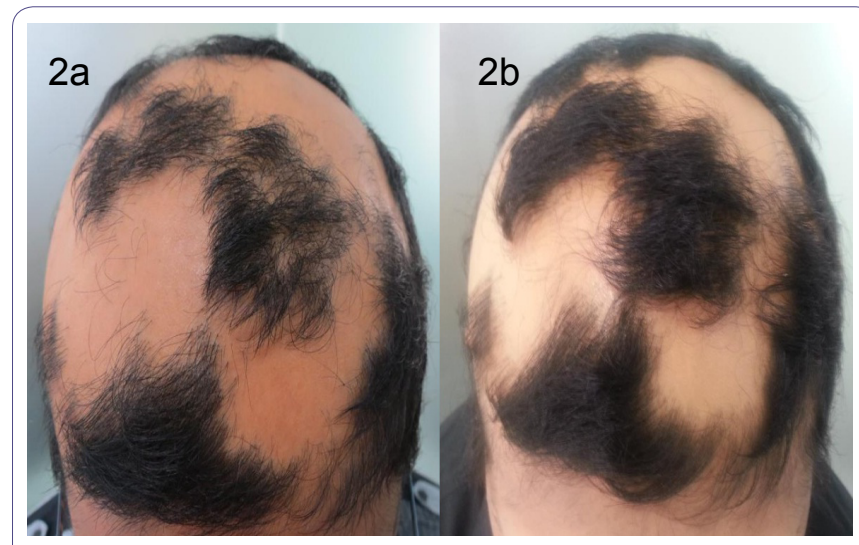

Figure 2: a) Patchy type: At the start of treatment; b) After 6 sessions

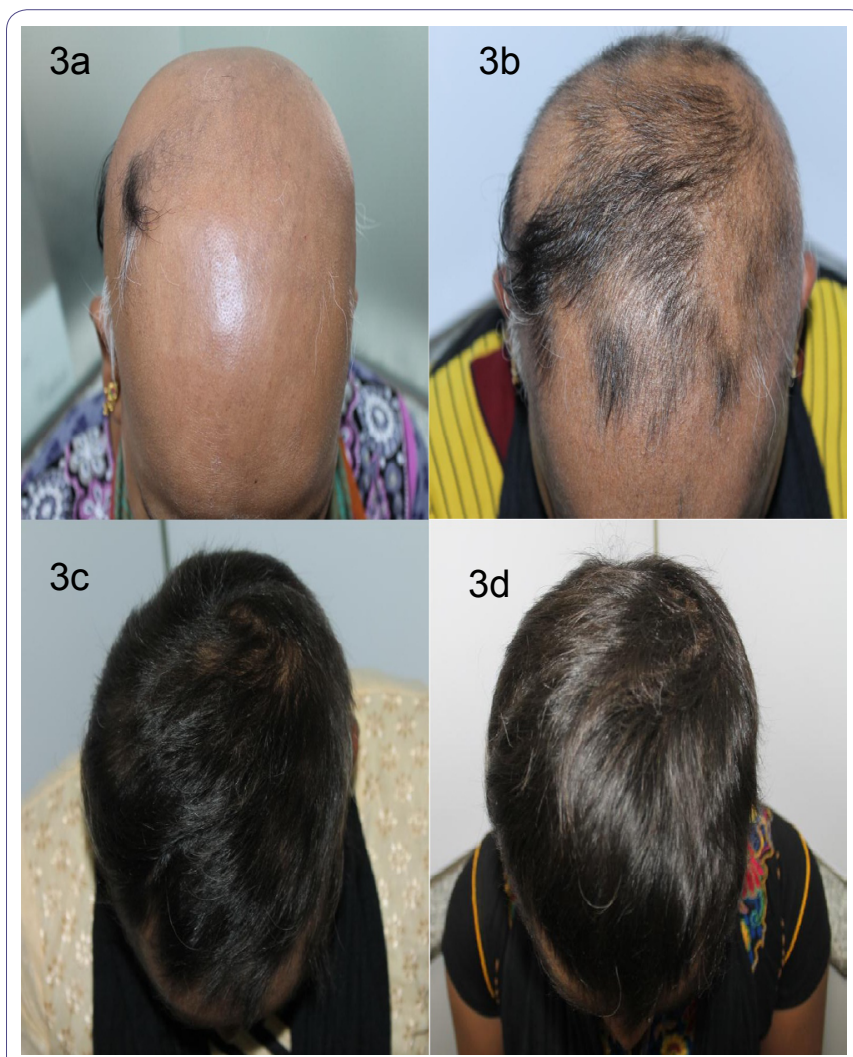

Figure 3: a) Subtotalispattern : At $1^{\text {st }}$ visit; b) After 3 sessions of TMA mesotherapy; c) After 6 sessions of TMA mesotherapy; d) 2 months after completion of 6 sessions and Maintenance with $2 \%$ Minoxidil

Clinically significant therapeutic response was observed in the ophiatis pattern and patchy type. The response in the totalis type and diffuse type was unremarkable except in 2 patients. There were no incidences of any adverse effects during or after the procedure. The demographics and results are tabulated in table 3.

\section{Discussion}

Definitive Treatment of alopecia areata continues to be challenging. Lack of a definite patho-mechanism or target molecule adds to the difficulty. Though a number of therapies have been tried with varied results in AA, there has been a significant percentage of relapse. Intralesional steroids, especially triamcinolone acetonide are often 


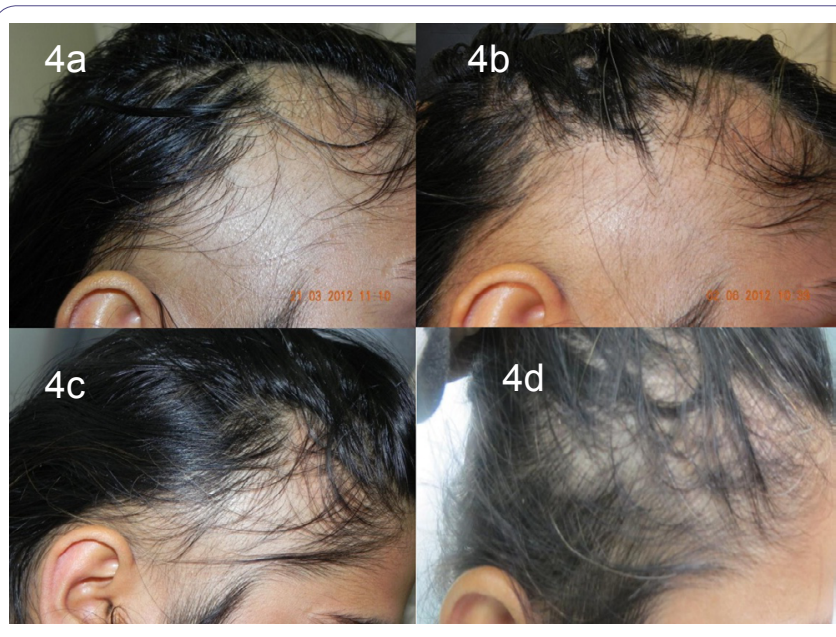

Figure 4: a) Ophiatic pattern : At 1st visit; b) No improvement after 3 months of conventional treatment; c) After 3 sessions of TMA mesotherapy;

d) 2 months after completion of 6 sessions healing, thus providing a new micro-environment for hair growth [9]. A recent study involving therapeutic wounding by microneedling in androgenetic alopecia also demonstrated a successful outcome [10]. In our study, we aim to reinforce the theory of therapeutic wounding in stimulation of hair follicle development.

\section{Conclusion}

Mesotherapy with triamcinolone acetonide $10 \mathrm{mg} / \mathrm{ml}$, delivered through dermaroller, appears to have a significant benefit in converting vellus hairs to terminal hair, without the worry of causing atrophy. It has potential to become a therapeutic modality of treatment of recalcitrant or relapsing cases of alopecia areata. To the best of our knowledge, this is the first case series employing a combination of therapeutic wounding and triamcinolone acetonide mesotherapy for treatment and maintenance of remission in alopecia areata, more so in cases of patchy type and ophiatic pattern. The drawback of this study is that there is no control group. Hence, placebo controlled randomized controlled trials are needed to establish the efficacy of therapeutic wounding with Triamcinolone Acetonide, over other methods of treatment of alopecia areata without involving wounding.

\begin{tabular}{|c|c|c|c|c|}
\hline Age of patient (in yrs) & Gender & Pattern of Alopecia Areata & $\begin{array}{l}\text { Clinician's assessment of improvement (0 to 2 } \\
\text { scale) }\end{array}$ & $\begin{array}{l}\text { Patient's assessment of improvement (0 to } \\
\text { 3 scale) }\end{array}$ \\
\hline 10 & M & Totalis & 1 & 1 \\
\hline 11 & M & Totalis & 0 & 0 \\
\hline 22 & M & Totalis & 1 & 2 \\
\hline 30 & M & Totalis & 2 & 3 \\
\hline 16 & F & Ophiasis & 2 & 2 \\
\hline 19 & F & Ophiasis & 2 & 2 \\
\hline 23 & M & Ophiasis & 1 & 2 \\
\hline 31 & M & Ophiasis & 1 & 1 \\
\hline 14 & M & Patchy & 1 & 2 \\
\hline 24 & M & Patchy & 2 & 2 \\
\hline 26 & M & Patchy & 1 & 1 \\
\hline 27 & F & Patchy & 2 & 2 \\
\hline 29 & M & Patchy & 2 & 1 \\
\hline 35 & F & Patchy & 1 & \\
\hline 26 & F & Diffuse & & \\
\hline
\end{tabular}

Table 3: Demographics and results.

used in the treatment of AA. However, while considering large patches of alopecia aerata, the injections can be painful and many times can lead to atrophy, especially if higher doses are used.

During microneedling therapy with dermaroller the needles pierce the stratum corneum and create microconduits (holes) without damaging the epidermis and leads to the release of growth factors and induces collagen synthesis [6]. The hair follicle is a reservoir for epithelial stem and progenitor cells, which reside in different niches [7]. Microneedling also stimulates the dermal papilla and stem cells [5].

The possibility that hair follicles develop de novo or a new following wounding was raised in few preclinical studies. Hair follicle regeneration and development has been shown to occur by over expression of Wnt ligand in the epidermis, after wounding in adult mice [8]. On wounding a cascade of events ensues, leading to release of epidermal and platelet derived growth factors, and over expression of fibroblast growth factors. Sun ZY et al., reported new hair growth around a healing wound in a man after a scald. They mentioned that after skin wounding occurs various signalling systems are setup to co-ordinate

\section{References}

1. Seetharam KA (2013) Alopecia Areata : An update. Indian J Dermatol Venereol Leprol 79: 563-575.

2. Ferrando J, Moreno-Arias GA (2000) Multi-injection plate for intralesional corticosteroid treatment of patchy alopecia areata. Dermatol Surg 26: 690-691.

3. Galán-Gutiérrez M, Rodríguez-Bujaldón A, Moreno-Giménez JC (2009) Update on the Treatment of Alopecia Areata. Actas Dermosifiliogr 100: 266-276.

4. Hordinsky MK (2011) Treatment of alopecia areata: "What is new on the horizon?". Dermatolo Ther 24: 364-368.

5. Jeonk K (2012) Repeated Microneedling stimulation induce the enhanced expression of Hair-growth-related-genes. Int J Trichol 4: 117.

6. Satish Doddaballapur (2009) Microneedling with Dermaroller. J Cutan Aesthet Surg 2: 110-111.

7. Jaks V, Kasper M, Toftgård R (2010) The hair follicle-a stem cell zoo. Exp Cell Res 316: 1422-1428.

8. Ito M, Yang Z, Andl T, Cui C, Kim N (2007) Wnt-dependent de novo hair follicle regeneration in adult mouse skin after wounding. Nature 447: 316-320. 
Citation: Chandrashekar BS, Sandeep MA, Vani Vasanth, Jagadish P, Rajashekar ML (2015) Triamcinolone Acetonide Mesotherapy in the Treatment of Recalcitrant Patches of Alopecia Areata - A Pilot Study. J Clin Dermatol Ther 2: 006.

- Page 4 of $4 \cdot$

9. Sun ZY, Diao JS, Guo SZ, Yin GQ (2009) A Very Rare Complication: New Hair Growth around Healing Wounds. J Int Med Res 37: 583-586.
10. Dhurat R, Sukesh M, Avhad G, Dandale A, Pal A, et al. (2013) A randomised evaluator blinded study of effect of microneedling in Androgenetic Alopecia: a pilot study. Int J Trichol 5: 6-11. 